\title{
Overexpression of $\mathrm{MPCl}$ inhibits the proliferation, migration, invasion, and stem cell-like properties of gastric cancer cells
}

This article was published in the following Dove Press journal:

OncoTargets and Therapy

24 October 2017

Number of times this article has been viewed

\author{
Xiang Zhou' \\ Zhu-juan Xiong ${ }^{2}$ \\ Shuo-meng Xiao' \\ Jin Zhou ${ }^{3}$ \\ Zhi Ding' \\ Ling-chao Tang' \\ Xiao-dong Chen' \\ Rui Xu' \\ Ping Zhao' \\ 'Department of Gastrointestinal \\ Surgery, Sichuan Cancer Hospital \& \\ Institute, Sichuan Cancer Center, \\ School of Medicine, University of \\ Electronic Science and Technology of \\ China, Chengdu, ${ }^{2}$ Nutritional Center, \\ Sichuan Cancer Hospital \& Institute, \\ Sichuan Cancer Center, School of \\ Medicine, University of Electronic \\ Science and Technology of China, \\ Chengdu, ${ }^{3}$ Department of Thoracic \\ Oncology, Sichuan Cancer Hospital \\ \& Institute, Sichuan Cancer Center, \\ School of Medicine, University of \\ Electronic Science and Technology of \\ China, Chengdu, China
}

Correspondence: Ping Zhao Department of Gastrointestinal Surgery, Sichuan Cancer Hospital \& Institute, Sichuan Cancer Center, School of Medicine, University of Electronic Science and Technology of China, 55th of 4 Section of People South Street, Chengdu 61004I, Sichuan Province, China

Tel +86 I89 08I 90029

Fax +862885420567

Email zhaopingzlyy@163.com
Abstract: Invasion and metastasis are major malignant characteristics of human gastric cancer (GC), but the molecular mechanisms underlying the invasion and metastasis of GC cells remain elusive. MPC1, a key factor that controls pyruvate transportation through the inner mitochondrial membrane, was reported to be downregulated and correlated with poor prognosis in several cancers. However, the effects of MPC1 on human GC have not been illustrated. In this study, we investigated the potential role of MPC1 in the proliferation, migration, invasion, and stem cell-like properties of human GC cells and evaluated its prognostic significance for patients with GC. We found that MPC1 protein and mRNA levels were significantly decreased in GC tissues and cell lines. Low MPC1 expression was associated with tumor T stage, $\mathrm{N}$ stage, and advanced tumor node metastasis stage. Decreased MPC1 expression was an independent prognostic marker and correlated with poor overall survival of patients with GC. Furthermore, overexpression of MPC1 inhibited the proliferation, migration, invasion, and stem cell-like properties of GC cells. These findings suggest that MPC1 may be a novel prognostic marker and a potential therapeutic target in human GC.

Keywords: gastric cancer, invasion, migration, MPC1, proliferation

\section{Introduction}

Gastric cancer (GC) is one of the most common malignant tumors of the digestive system and the third most common cause of cancer-related death. ${ }^{1}$ Although the mortality rate has been decreasing in recent years thanks to significant improvements in early detection, surgical techniques, and chemotherapy, the clinical outcomes for patients with advanced-stage GC are unfavorable. Currently, accumulating evidence suggests that mitochondrial metabolism is required for tumorigenesis. ${ }^{2}$ It has been shown that mitochondrial respiration plays a critical role in treatment-resistant cancer cells, ${ }^{3}$ metastatic and circulating cancer cells, ${ }^{4}$ cancer stem cells, and tumor-initiating cells within several cancers. ${ }^{5}$ Moreover, key metabolic enzymes and pathways associated with mitochondrial metabolism are reported to be closely correlated with tumor initiation and progression. ${ }^{6}$

MPC1, an approximately $12 \mathrm{kDa}$ protein with two predicted transmembrane domains, is located in the inner mitochondrial membrane. MPC1 was recently identified to be a critical component of a transporter complex to control rate-limiting pyruvate transportation through the inner mitochondrial membrane. ${ }^{78}$ Loss of MPC1 protein results in disability of the mitochondrial pyruvate carrier (MPC) complex function and blocks pyruvate entry into the tricarboxylic acid cycle, leading to a metabolism switch to increase glycolysis and the compensatory usage of glutamine. ${ }^{9,10}$ 
Currently, the important role of MPC1 was unveiled in several diseases, in particular in solid tumors. ${ }^{11,12}$ Decreased expression of MPC1 has been correlated with poor survival in esophageal squamous cell carcinoma, and lung, colon, and prostate cancers. ${ }^{13-16}$ It was also discovered that treatment with the MPC inhibitor UK5099 upregulated the expression of the stem cell markers Oct $3 / 4$ and Nanog in prostate cancer cells. ${ }^{12}$ However, the clinical significance and function of MPC1 in GC have not been established.

In the present study, we report that the protein and mRNA levels of MPC1 are downregulated in both human GC tissues and cell lines. Decreased MPC1 expression is associated with tumor invasion depth (T stage), lymph node metastasis (N stage), and advanced tumor node metastasis (TNM) stage. Low MPC1 expression is significantly correlated with poor overall survival in patients with GC. Overexpression of MPC1 inhibited the proliferation, migration, invasion, and stem cell-like properties of GC cells. Our results suggest that MPC1 may be considered as a novel prognostic indicator and a therapeutic target for human GC.

\section{Materials and methods Cell culture}

Human GC cell lines MGC803, BGC823, SGC7901, and AGS (Cell Bank of Shanghai Institute of Cell Biology, Chinese Academy of Sciences, Beijing, China) as well as the immortalized gastric epithelium cell line GES-1 (American Type Culture Collection, Manassas, VA, USA) were cultured in RPMI 1640 medium (Sigma-Aldrich Co., St Louis, MO, USA) supplemented with 10\% FBS (Sigma-Aldrich Co.).

\section{Patients and tissue samples}

From January 2003 to December 2009, we collected a total of 152 formalin-fixed and paraffin-embedded surgical specimens of GC tumors and paired adjacent normal tissues from patients with GC who underwent surgical resection at the Sichuan Cancer Hospital \& Institute, Chengdu, Sichuan Province, China. All patient samples were adenocarcinoma. All the 152 patients were treated with radical gastrectomy and D2 lymphadenectomy. The postoperative chemotherapy was based on fluorouracil regimens and none of the subjects received preoperative chemotherapy. All clinical information from the 152 specimens in this project was classified according to different clinical features (Table S1). All patients were followed-up more than 5 years. Written informed consent was obtained from all patients. The study was approved by the Ethical Committee of Sichuan Cancer Hospital \& Institute.
Immunohistochemistry (IHC) and scoring IHC detection of MPC1 was performed on $4 \mu \mathrm{m}$ tissue sections with Dako Real Envision Detection System (Dako Denmark A/S, Glostrup, Denmark) according to the manufacturer's instructions. After antigen retrieval and blocking, the tissue slides were incubated with the mouse anti-MPC1 monoclonal antibody (1:300; Abcam, Cambridge, UK) at $4^{\circ} \mathrm{C}$ overnight. Next, the slides were incubated at $37^{\circ} \mathrm{C}$ for $30 \mathrm{~min}$ after an HRP-conjugated secondary antibody (Dako Denmark A/S) was added. Each GC specimen was histologically graded by two pathologists according to the 7th edition of the American Joint Committee on Cancer TNM classification system ${ }^{17}$ in a double-blinded manner. H\&E staining was performed to confirm the GC characteristics of the tumor sections (Figure S1A). The percentage of positive cancer cells was scored as: $1,1 \%$ to $25 \%$ positive cells; $2,26 \%$ to $50 \%$ positive cells; $3,51 \%$ to $75 \%$ positive cells; and $4,>75 \%$ positive cells. The intensity was scored as follows: 0 , no staining cells; 1 , weak staining; 2, mild staining; and 3, strong staining (Figure S1B). The IHC staining score was obtained by multiplying the staining intensity from the average percentage of positive cells, following an accepted semi-quantitative evaluation method. ${ }^{18}$ Scores were analyzed using the statistical software X-tile ${ }^{19}$ with a score of 6 as the cutoff value. Therefore, total scores $\geq 8$ were defined as $\mathrm{MPC} 1^{\text {high }}$ and scores $\leq 6$ were defined as MPC $1^{\text {low }}$ (Figure S1C).

\section{Western blotting}

Cells and tissue samples were lysed in RIPA lysis buffer (Beyotime, Jiangsu, China) supplemented with protease inhibitors (phenylmethylsulfonyl fluoride [PMSF]; Thermo Fisher Scientific, Waltham, MA, USA) on ice. Lysates were centrifuged at $12,000 \mathrm{~g}$ for $15 \mathrm{~min}$ at $4^{\circ} \mathrm{C}$ before aliquots of $20 \mu \mathrm{g}$ proteins were electrophoresed on 15\% SDS-PAGE gels (Bio-Rad Laboratories Inc., Hercules, CA, USA), transferred onto PVDF membranes (EMD Millipore, Billerica, MA, USA), and incubated overnight with primary antibodies: anti-MPC1 (Abcam, 1:500), anti-FLAG (Beyotime, 1:1,000), anti-Sox-2 (Abcam, 1:500), anti-Oct-4 (Abcam, 1:400), anti-Nanog (Abcam, 1:300), and anti- $\beta$-actin (Beyotime, 1:1,000). The membranes were then incubated with appropriate HRP-conjugated secondary antibody for $2 \mathrm{~h}$ at room temperature. Chemiluminescence was detected using SuperSignal West Femto Maximum Sensitivity Substrate (enhanced chemiluminescence, Thermo Fisher Scientific). $\beta$-actin was used as a loading control. All of the experiments were performed in triplicate.

\section{RNA extraction and qRT-PCR}

Total RNA was extracted from cells and 15 pairs of frozen tissue specimens using Trizol reagent (Takara Bio, Shiga, 
Japan) according to the manufacturer's instructions. Reverse transcription was performed using reverse transcription reagents (Takara). The resulting cDNA was subjected to quantitative reverse transcription polymerase chain reaction (qRT-PCR) using the CFX96 Real-Time Quantitative PCR system (Bio-Rad Laboratories Inc.) with SYBR Premix Ex Taq II (Takara) following the manufacturer's instructions. The primers used in this study are listed in Table S2. The relative mRNA expression levels were determined by the cycle threshold $(\mathrm{Ct})$ normalized against $\beta$-actin using the $2^{-\Delta \Delta \mathrm{Ct}}$ formula. Experiments were performed in triplicate.

\section{NCBI GEO datasets analysis}

The mRNA levels of MPC1 in GC are publically available from the NCBI GEO database (accession number: GSE27342, GSE26942). Data were log2 transformed and quantile normalized using the R software (version 3.2.5; R Foundation for Statistical Computing, Vienna, Austria) along with packages from the BioConductor project as previously described. ${ }^{20}$ For the survival analysis of MPC1, an online tool was used to conduct Kaplan-Meier survival analysis, which included 380 patients with GC after surgery with available clinical data. The analyzed data were downloaded from the website and then Kaplan-Meier curves were plotted using GraphPad Prism 6.01 software (GraphPad Software, Inc., La Jolla, CA, USA). For the expression of the genes, each percentile of expression between the lower and upper quartiles was computed and the best performing threshold was used as the final cutoff for the univariate Cox regression analysis. The hazard ratio with $95 \%$ confidence interval and $P$-value were calculated.

\section{Overexpression of $\mathrm{MPCl}$ using lentivirus}

To investigate the function of MPC1 in GC cells, we overexpressed MPC1 in MGC803 and SGC7901 cells. Lentiviral particles containing the GV341 expression vector were performed by GeneChem (Shanghai, China). The amplified sequences were C-terminally fused to FLAG (DYKDDDDK) tags by PCR. Lentiviral particles with a blank vector were used as a negative control. Next, the lentiviral-MPC1FLAG and control lentivirus were infected into SGC7901 and MGC803 cells for $24 \mathrm{~h}$ in medium containing $6 \mu \mathrm{g} /$ $\mathrm{mL}$ polybrene (Sigma-Aldrich Co.). Fresh culture medium containing $4 \mu \mathrm{g} / \mathrm{mL}$ puromycin was added to select stable puromycin-resistant GC cells.

\section{Colony formation assay}

To analyze differences in colony formation after MPC1 overexpression, SGC7901 and MGC803 GC cells were thoroughly dissociated and then plated in 6-well plates at a density of 500 cells per well in triplicate. Cells were cultured in $2 \mathrm{~mL}$ RPMI 1640 medium containing 10\% FBS. The plates were then incubated for 14 days at $37^{\circ} \mathrm{C}$ with $5 \% \mathrm{CO}_{2}$ until most cell clones had generated more than 50 cells. After staining with Giemsa dye for $15 \mathrm{~min}$ at room temperature, the numbers of colonies containing more than 50 cells were counted. Experiments were repeated three times.

\section{Cell proliferation assay}

Cells dissociated from SGC7901 and MGC803 were prepared into single cell suspension and seeded in 96-well plates at approximately 1,000 cells per well in $0.1 \mathrm{~mL}$ of RPMI 1640 medium containing 10\% FBS. At 24, 48, 72, and 96 h, culture medium was abandoned and then $0.1 \mathrm{~mL}$ of fresh RPMI 1640 containing 10\% FBS and $0.01 \mathrm{~mL}$ cell counting kit-8 solution (CCK-8; Dojindo Laboratories, Tokyo, Japan) was added. After incubation at $37^{\circ} \mathrm{C}$ for $1.5 \mathrm{~h}$, the plates were agitated for $15 \mathrm{~min}$, and the optical density of the solution was measured at $450 \mathrm{~nm}$ in a photometer according to the protocol.

\section{Migration and invasion assays}

Cell migration and invasion assays were performed using modified Boyden chambers with $8 \mathrm{~mm}$ pore filter inserts in 24-well plates (BD Biosciences, San Jose, CA, USA). For the invasion assay, the insert membranes were precoated with $10 \mu \mathrm{L}$ of Matrigel (BD Biosciences)/RPMI 1640 (1:1, v/v) for $30 \mathrm{~min}$ at $37^{\circ} \mathrm{C}$. GC cells were serum-starved for $12 \mathrm{~h}$ before being added to the upper chamber at a density of $2 \times 10^{4}$ cells per well with $200 \mu \mathrm{L}$ serum-free RPMI 1640 medium. Then, $500 \mu \mathrm{L}$ RPMI 1640 medium containing 10\% FBS was added to the basal chambers. The plates were incubated for $24 \mathrm{~h}$. For the migration assay, the insert membranes were not coated with Matrigel, but they were cultured under the same conditions. After fixation with 4\% paraformaldehyde for $20 \mathrm{~min}$, the insert membranes were cut and the cells on the upper surface of the membrane were removed with a cotton swab. Then the cells on the lower surface of the membrane were stained with crystal violet solution (Beyotime). The number of cells attached to the lower surface was counted in five randomly selected high-power fields under an inverted microscope and photographed.

\section{Tumorsphere formation assay}

Adherent SGC7901 and MGC803 cells with different treatments were harvested by trypsin digestion and suspended in serum-free DMEM/F12 medium (Gibco, Thermo Fisher Scientific) supplemented with $20 \mathrm{ng} / \mathrm{mL}$ recombinant human EGF (Sigma-Aldrich Co.), 20 ng/mL bFGF 
(Sigma-Aldrich Co.), and 1× B27 (Invitrogen, Thermo Fisher Scientific). The cells were seeded in 24-well ultralow-attachment plates (Corning Incorporated, Corning, NY, USA) to acquire tumorspheres. The number of primary GC cell spheres was counted after 2 weeks in five random fields. Each experiment was performed in triplicate.

\section{Subcutaneous xenograft tumorigenicity experiments}

This experiment was conducted in accordance with Animal Welfare and 3Rs (Replacement, Reduction, and Refinement) guidelines and was approved by the Ethical Committee of Sichuan Cancer Hospital \& Institute. Female BALB/c nude mice ( 5 to 6 weeks old) were purchased from Laboratory Animal Center of Sichuan University (Chengdu, China) and housed in a pathogen-free environment. MPC1-FLAG or control SGC7901 cells were injected subcutaneously into the axilla of the mice $\left(2 \times 10^{4}\right.$ cells in $0.2 \mathrm{~mL}$ Matrigel [1:1, v/v]). Subcutaneous tumor growth was inspected every day. The mice were sacrificed 4 weeks after implantation, and the tumors were collected and weighed.

\section{Statistical analysis}

All experiments were conducted at least three times. Results were presented as the mean \pm standard deviation. Statistical analysis was performed using the SPSS 19.0 software (IBM Corporation, Armonk, NY, USA). Student's $t$-test was used for comparing the two groups and Pearson $\chi^{2}$ test was used to determine the correlation between MPC1 expression and the clinicopathological features of patients with GC. The mRNA levels of MPC1 in GSE27342 were compared using paired $t$-test. Kaplan-Meier survival plots and log-rank statistics were used to compare the survival rates of patients. Univariate and multivariate survival analyses were performed using a Cox proportional hazards model with stepwise selection. The cutoff value of the IHC score was determined by the statistical software X-tile. ${ }^{19}$ A value of $P<0.05$ was considered significant.

\section{Results}

\section{$\mathrm{MPCI}$ protein and mRNA levels were downregulated in both GC tissues and cell lines}

We detected the expression of MPC1 in our 152 paired tumor tissues and adjacent normal tissues. MPC1 staining was observed in the cytoplasm of GC cells (Figure 1A). The proportion of MPC $1{ }^{\text {high }}$ cells in GC tissues $(48.68 \%, 74 / 152)$ was markedly lower than that in adjacent normal tissues
(79.61\%, 121/152, $P<0.001)$ (Table 1). Moreover, the IHC scores of MPC1 in GC tissues were significantly lower than the corresponding normal tissues $(P<0.001$, Figure 1B), indicating that the protein levels of MPC1 were significantly downregulated in GC tissues. We detected the mRNA levels of 15 pairs of fresh GC tissues and corresponding adjacent normal tissues by qRT-PCR analysis. The results demonstrated that MPC1 mRNA levels were higher in normal specimens than in GC tissues $(P<0.01$, Figure $1 C)$. In addition, results from GSE27342 and GSE26942 demonstrated that the mRNA levels of MPC1 in GC tissues were obviously lower than in adjacent normal tissues $(P<0.001$ and $P=0.0016$, respectively, Figure 1D). We then analyzed two pairs of fresh GC tissues and corresponding adjacent normal tissues by Western blotting and our results showed that the protein levels of MPC1 in GC tissues were downregulated (Figure 1E). To investigate the expression of MPC1 protein and mRNA levels in GC cell lines in comparison with gastric epithelium cells, MGC803, SGC7901, BGC823, AGS, and GES-1 were tested. The results demonstrated that the MPC1 protein and mRNA levels were obviously downregulated in four GC cells lines (MGC803, SGC7901, BGC823, and AGS) compared with gastric epithelium cell line GES-1, especially MGB803 and SGC7901 (Figure 1F and G). As a result, MGC803 and SGC7901 were employed for our further study. Overall, our results suggested that the MPC1 protein and mRNA levels were downregulated in both GC tissues and cell lines.

\section{$\mathrm{MPCl}$ expression was correlated with the clinicopathological features of patients with GC}

We analyzed the correlation between MPC1 expression and the clinicopathological features of patients with GC. As shown in Table 2, we listed the clinicopathological indices of 152 patients with GC and corresponding MPC1 levels. Among the 152 cases, 78 cases were $\mathrm{MPC}^{\text {low }}$ and 74 were MPC $1^{\text {high }}$ (Table 1). MPC1 expression was negatively correlated with $\mathrm{T}$ stage (tumor invasion depth) $(P<0.001$, Table 2; Figure 2A), N stage (lymph node metastasis) $(P=0.037)$, and TNM stage $(P=0.028)$. No significant correlations between MPC1 expression and age, sex, tumor site or histological grade were found (Table 2). Furthermore, we analyzed the relationship between IHC scores of MPC1 and TNM stage in our 152 cases and our results revealed that low MPC1 was correlated with advanced TNM stage $(P=0.005$, Figure $2 \mathrm{~B})$. We also analyzed the mRNA levels of MPC1 in different TNM stages from GSE26942. Results from GSE26942 indicated that lower MPC1 expression was 

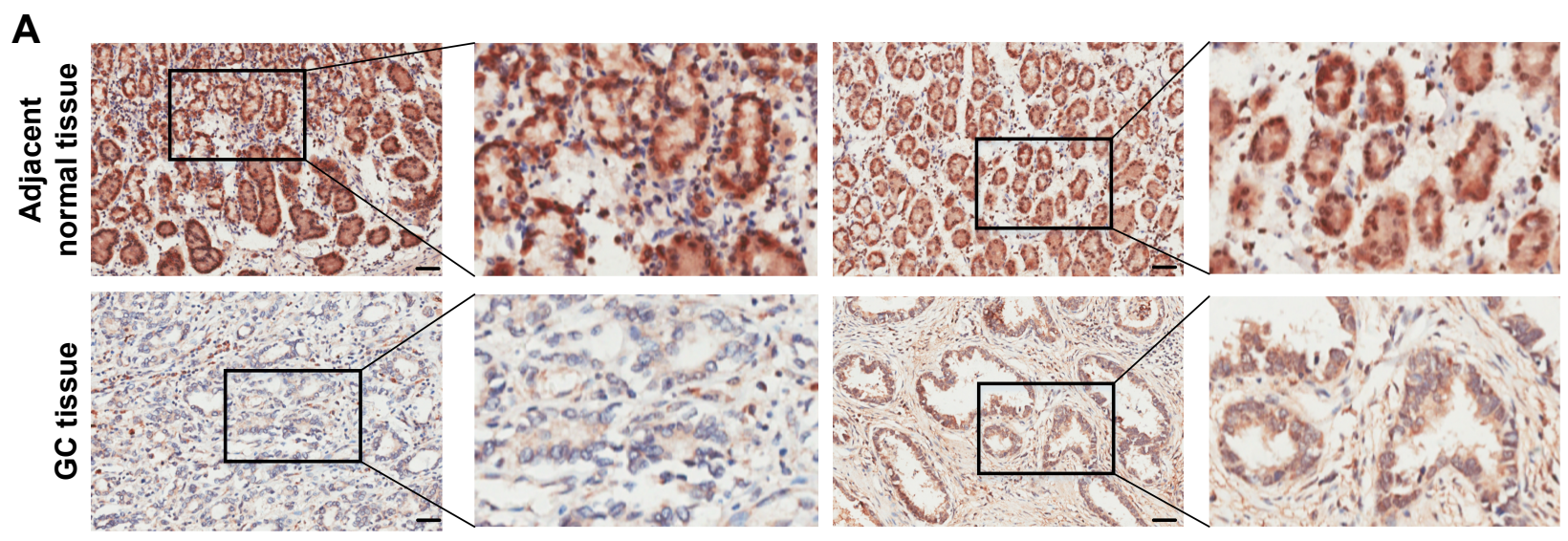

B

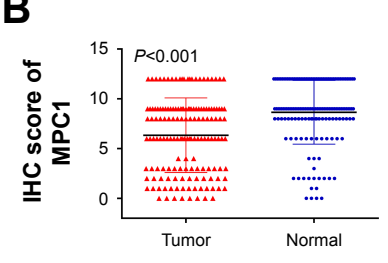

C

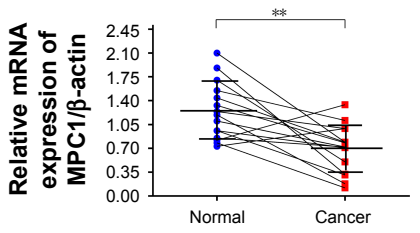

D

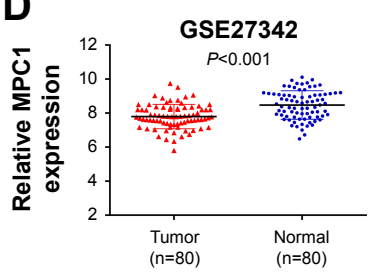

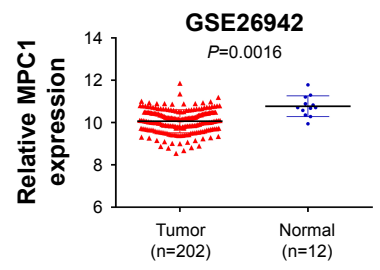

GSE26942
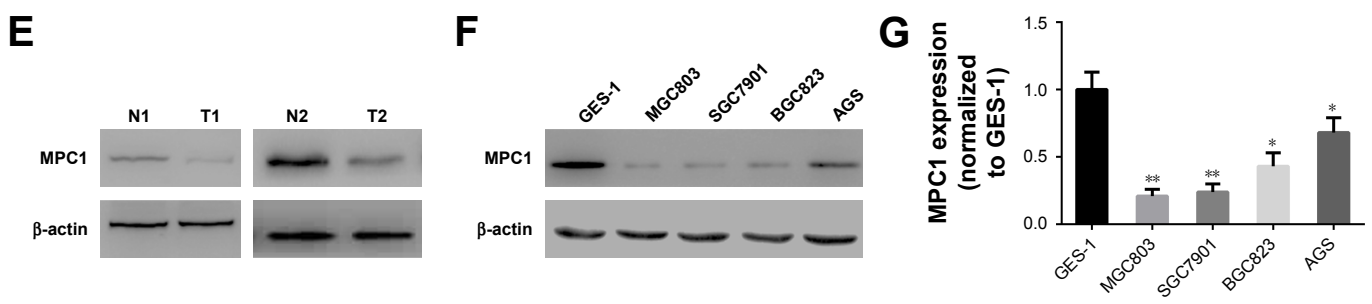

Figure I The protein and mRNA levels of MPCI were significantly decreased in GC tissues and cell lines.

Notes: (A) Representative images of immunohistochemistry (IHC) staining of MPCI in GC and adjacent normal tissues. Scale bar, $50 \mu \mathrm{m}$. (B) IHC scores of MPCI of adjacent normal tissues and cancerous tissues in I52 paired GC specimens $(P<0.00 \mathrm{I})$. (C) MPCI mRNA levels in I5 pairs of fresh GC specimens and adjacent normal tissues $(P<0.01)$. (D) Analyses of MPCI mRNA levels in GSE27342 and GSE26942 ( $P<0.00 \mathrm{I}, P=0.00 \mathrm{I}$, respectively). (E) Western blotting analysis of MPCI in two pairs of GC and adjacent normal tissues. (F) Detection of MPCI in GC cell lines (SGC790I, MGC803, BGC823, and AGS) and gastric epithelium cell line (GES-I) by Western blotting analysis. (G) Detection of MPCI in GC cell lines (SGC790I, MGC803, BGC823, and AGS) and GES-I by qRT-PCR analysis. $* P<0.05, * * P<0.01$.

Abbreviations: GC, gastric cancer; IHC, immunohistochemistry.

correlated with advanced TNM stages $(P=0.016$, Figure $2 \mathrm{C})$. These results suggested that decreased MPC1 expression was significantly associated with tumor invasion depth, lymph node metastasis, and TNM stage in patients with GC.

\section{Decreased MPCI expression predicted poor overall survival of patients with GC} To further evaluate the prognostic significance of MPC1 expression in GC, Kaplan-Meier survival analysis of our 152 patients with GC were conducted. The overall survival

Table I MPCI expression in GC and adjacent normal mucosa

\begin{tabular}{lllll}
\hline Tissue type & MPCI Iow $^{\text {MPCI }}$ & Migh $^{2}$ & $\chi^{2}$ & -value \\
\hline Carcinoma & 78 & 74 & 31.594 & 0.000 \\
Adjacent normal mucosa & $3 \mathrm{I}$ & 121 & & \\
\hline
\end{tabular}

Abbreviation: GC, gastric cancer. rates for $\mathrm{MPC} 1^{\text {high }}$ patients were significantly higher than those with MPC $1^{\text {low }}$ tumors $(P<0.001$, Figure $3 A)$. Univariate and multivariate analyses of the 152 cases showed that low MPC1 expression was an independent prognostic indicator for the overall survival of GC patients (Table 3). Moreover, survival analysis of $380 \mathrm{GC}$ patients after surgery from the NCBI GEO database using the online tool kmplot (http://www.kmplot.com/gastric) indicated that low MPC1 expression was associated with poor overall survival $(P=0.0036$, Figure 3B).

\section{Overexpression of $\mathrm{MPCl}$ attenuated the proliferation, migration, and invasion of GC cells}

To confirm the function of MPC1 in GC progression, we evaluated the subsequent effects of MPC1 overexpression on 
Table 2 The relationship between MPCI expression and clinicopathological features of GC patients

\begin{tabular}{|c|c|c|c|c|}
\hline \multirow{2}{*}{$\begin{array}{l}\text { Clinicopathological } \\
\text { features }\end{array}$} & \multirow[t]{2}{*}{$\mathbf{N}$} & \multicolumn{3}{|c|}{ MPCI } \\
\hline & & Low & High & $P$-value \\
\hline Age (years) & & & & 0.688 \\
\hline$\geq 60$ & 60 & 32 & 28 & \\
\hline$<60$ & 92 & 46 & 46 & \\
\hline Sex & & & & 0.257 \\
\hline Male & 100 & 48 & 52 & \\
\hline Female & 52 & 30 & 22 & \\
\hline Tumor site & & & & 0.120 \\
\hline Proximal gastric & 61 & 36 & 25 & \\
\hline Distal gastric & 91 & 42 & 49 & \\
\hline Histological grade & & & & 0.486 \\
\hline $\mathrm{GI}+\mathrm{G} 2$ & 43 & 24 & 19 & \\
\hline G3 & 109 & 54 & 55 & \\
\hline T stage & & & & 0.000 \\
\hline $\mathrm{TI}-\mathrm{T} 2$ & 49 & 13 & 36 & \\
\hline T3-T4 & 103 & 65 & 38 & \\
\hline $\mathrm{N}$ stage & & & & 0.037 \\
\hline No & 61 & 25 & 36 & \\
\hline NI-N3 & 91 & 53 & 38 & \\
\hline TNM stage & & & & 0.028 \\
\hline $\mathrm{I} / \mathrm{II}$ & 89 & 39 & 50 & \\
\hline III/IV & 63 & 39 & 24 & \\
\hline
\end{tabular}

Abbreviations: GC, gastric cancer; TNM, tumor node metastasis.

the proliferation, migration, and invasion of GC cells. Overexpression of MPC1 protein levels in MGC803 and SGC7901 cells was tested by Western blotting analysis (Figure 4A). The colony formation assay showed that the number and average size of colonies formed by MPC1-FLAG MGC803 and SGC7901 cells in vitro were smaller than those formed by control cells $(P<0.05$, Figure $4 \mathrm{~B}$ and $\mathrm{C})$. In our CCK-8 assays, the proliferation ability was significantly inhibited in MPC1-FLAG MGC803 and SGC7901 cells (Figure 4D). The migration and invasion assays showed that overexpression of MPC1 significantly impaired the migrating and invasive capabilities of GC cells $(P<0.05$, Figure 4E and F). These results suggested that overexpression of MPC1 inhibited the proliferation, migration, and invasion of GC cells in vitro.

\section{Overexpression of $\mathrm{MPCl}$ impaired stem cell-like properties in GC cells}

To investigate whether overexpression of MPC1 has an effect on stem cell properties of GC cells, stem cell markers such as Nanog, Sox-2, and Oct-4 ${ }^{21,22}$ were detected using Western blotting and qRT-PCR analyses. Enhanced MPC1 expression led to significant decrease in the protein and mRNA levels of Nanog, Sox-2, and Oct-4 compared with control cells (Figure 5A and B). We then evaluated the effect of MPC1 overexpression on the sphere formation ability of GC cells. We found that the number and size of spheres formed by MPC1-FLAG cells were decreased (Figure 5C and D). In vivo, tumor-initiating capability of SGC7901 cells was inhibited by MPC1 overexpression. The size of xenograft tumors derived from MPC1-FLAG cells was significantly smaller than those formed by control cells (Figure 5E).
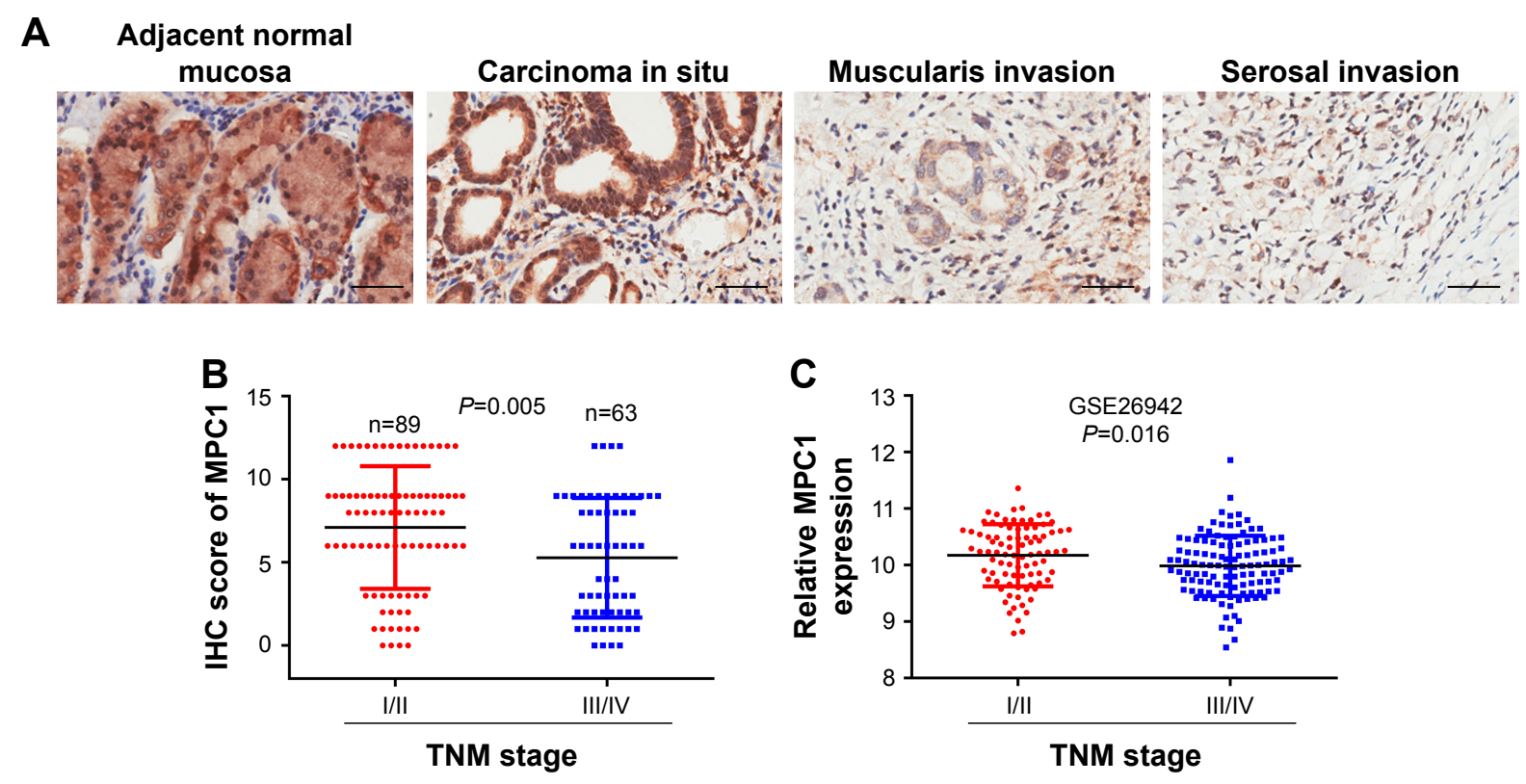

Figure $2 \mathrm{MPCl}$ expression was closely correlated with TNM stage in patients with GC.

Notes: (A) Representative images of MPCI staining in normal gastric mucosa and GC samples at different infiltration depths. Scale bar, $50 \mu$ m; magnification $\times 200$. (B) IHC scores of $\mathrm{MPCI}$ in patients with TNM III/IV tumors were significantly lower compared with TNM I/II tumors ( $P=0.005)$. (C) Analysis of NCBI GEO dataset GSE26942 showed that the mRNA levels of MPCI in TNM III/IV cases were obviously lower than those of TNM I/II cases $(P=0.016)$.

Abbreviations: TNM, tumor node metastasis; GC, gastric cancer; IHC, immunohistochemistry. 

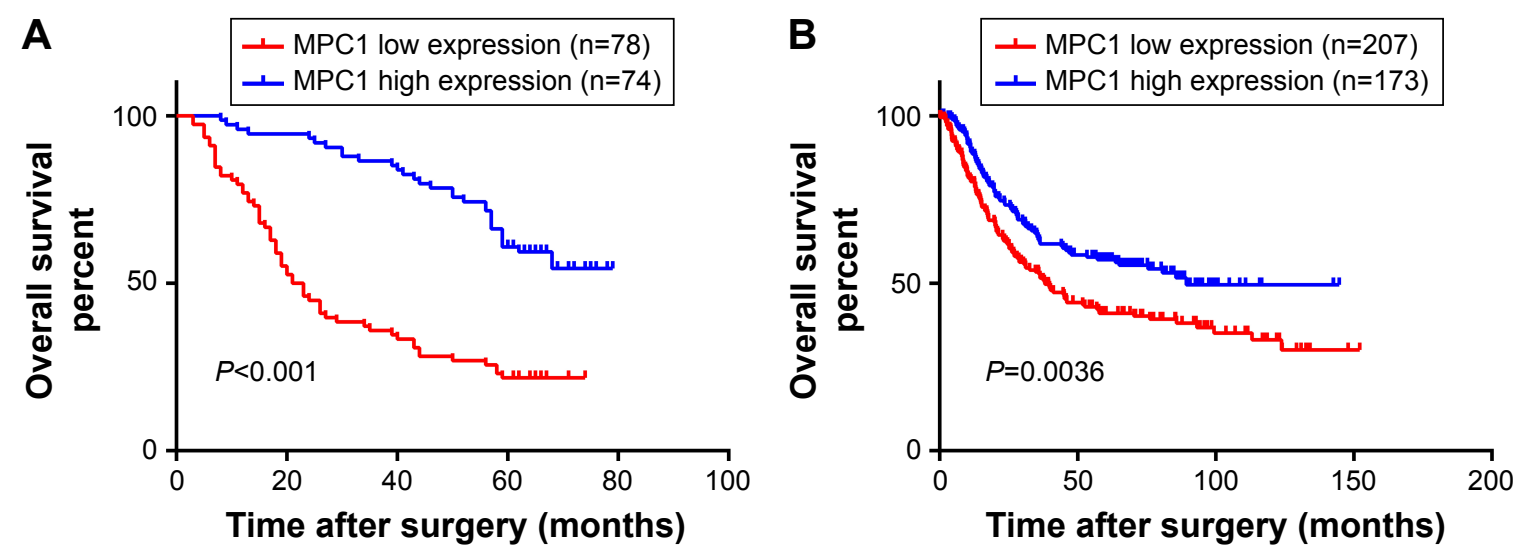

Figure 3 Kaplan-Meier analysis of overall survival according to the expression of MPCI in patients with GC.

Notes: (A) Kaplan-Meier analyses of 152 patients with GC showing MPCl high patients $(n=74)$ have longer overall survival as compared with MPCIlow patients ( $\mathrm{n}=78$ ) $(P<0.00$ I). (B) Kaplan-Meier analyses of $380 \mathrm{GC}$ patients from NCBI GEO database showing that the overall survival rates for MPCI high patients ( $\mathrm{n}=\mathrm{I} / 3)$ are significantly higher than MPCI low patients $(n=207, P=0.0036)$.

Abbreviation: GC, gastric cancer.

The weights of the xenograft tumors corresponded to their sizes $(P<0.01$, Figure 5F).

\section{Discussion}

GC cells have metabolic alterations characterized by an increased rate of glycolysis and producing high amounts of lactate in the presence of oxygen, a process known as the Warburg effect. ${ }^{23,24}$ Multiple mechanisms contribute to this metabolic derangement in cancer cells, of which the metabolism of pyruvate is one of the most important differences made by normal cells and cancer cells. Cancer cells divert pyruvate and its precursors to fuel other anabolic processes or convert it to lactate for excretion..$^{25}$ Altered pyruvate metabolism appears to be critical in enabling and promoting the transformed phenotype in many cancers. One of the mechanisms to explain the decrease in mitochondrial pyruvate oxidation within cancer cells was a loss of mitochondrial pyruvate import. ${ }^{26}$

MPC1 and MPC2 were originally known as BRP44L and BRP44, and were recently found to form a hetero-oligomeric complex mounting in the inner mitochondrial membrane and facilitating pyruvate into the mitochondrial matrix. ${ }^{7,8}$ Downregulation of MPC1 was reported in a variety of tumor cell lines and solid tumors. ${ }^{15}$ Several studies illustrated that cancer cells could reduce the activity of MPC by inhibiting the expression of MPC1 to facilitate tumor growth, and that tumor metastasis could be impaired by enhancing MPC1 expression. ${ }^{14,27}$ In prostate cancer, it was discovered that COUP-TF II was recruited to the MPC1 promoter to suppress MPC1 expression and overexpression of MPC1 suppressed tumor cell growth and invasion. ${ }^{14}$ Although studies demonstrated that MPC1 played a critical role in several cancers, the function of MPC1 in GC cells and its prognostic significance in patients with $\mathrm{GC}$ remained ambiguous.

In this study, we firstly detected the protein and mRNA levels of MPC1 in GC tissues and cell lines with Western blotting, immunohistochemical staining, and qRT-PCR analyses. Furthermore, mRNA levels of MPC1 in two NCBI GEO datasets (accession numbers: GSE27342 and GSE26942) were explored and a publicly available GC patients database (NCBI GEO database) was analyzed using an online tool (http://www.kmplot.com/gastric) to generate

Table 3 Univariate and multivariate analyses of overall survival in GC patients

\begin{tabular}{|c|c|c|c|c|}
\hline \multirow{2}{*}{$\begin{array}{l}\text { Prognostic } \\
\text { variables }\end{array}$} & \multicolumn{2}{|l|}{ Univariate analysis } & \multicolumn{2}{|c|}{ Multivariate analysis } \\
\hline & HR (95\% Cl) & $P$-value & HR (95\% Cl) & $P$-value \\
\hline Age (years) & I.243 (0.819-I.886) & 0.307 & I.I89 (0.764-I.852) & $0.48 I$ \\
\hline Sex & $1.022(0.667-1.565)$ & 0.921 & $0.984(0.630-1.538)$ & 0.842 \\
\hline Histological grade & $0.698(0.45 I-1.1080)$ & 0.106 & $0.733(0.466-1.154)$ & 0.131 \\
\hline T stage & $2.387(1.463-3.895)$ & 0.000 & $1.279(0.703-2.325)$ & 0.709 \\
\hline $\mathrm{N}$ stage & $2.218(1.420-3.464)$ & 0.000 & $2.034(1.300-3.183)$ & 0.069 \\
\hline TNM stage & $2.200(1.456-3.325)$ & 0.000 & $1.037(0.607-1.770)$ & 0.462 \\
\hline Low MPCI expression & $0.285(0.184-0.442)$ & 0.000 & $0.268(0.172-0.419)$ & 0.000 \\
\hline
\end{tabular}

Abbreviations: GC, gastric cancer; $\mathrm{HR}$, hazard ratio; $\mathrm{Cl}$, confidence interval; TNM, tumor node metastasis. 
Kaplan-Meier curves. We found that the protein and mRNA levels of MPC1 were downregulated in both GC tissues and cell lines. Our results expanded the knowledge of the expression patterns of MPC1, which were also reported to be downregulated in esophageal squamous cell carcinoma and prostate, colon, kidney, and lung cancers. ${ }^{15,16,28}$ Our survival analysis demonstrated that low MPC1 expression was an independent prognostic indicator and predicted poor overall survival in patients with GC. To our knowledge, this is the first clinicopathological study to link MPC1 to the clinical outcome of patients with GC.

Considering that aerobic glycolysis has been generally associated with cancer cell proliferation, we evaluated the function of MPC1 in the proliferation of GC cells. Through colony
A

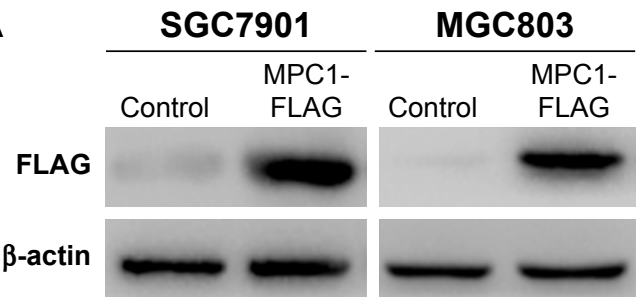

C

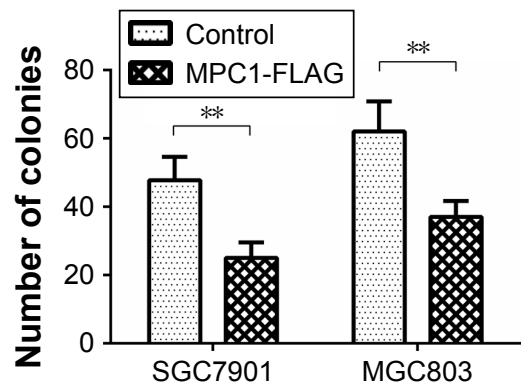

D

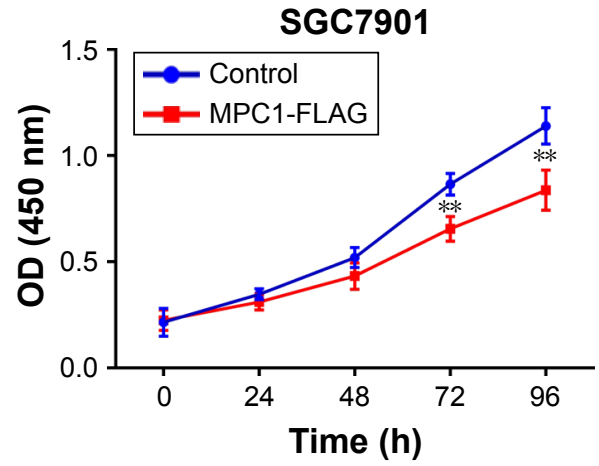

E

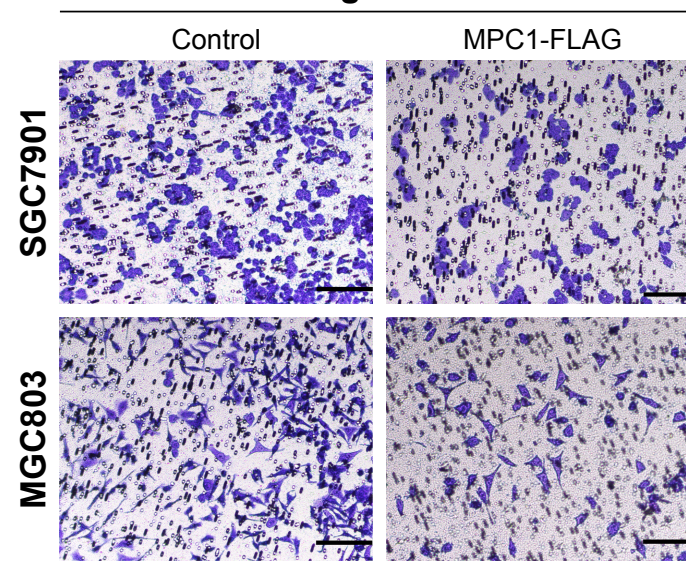

B
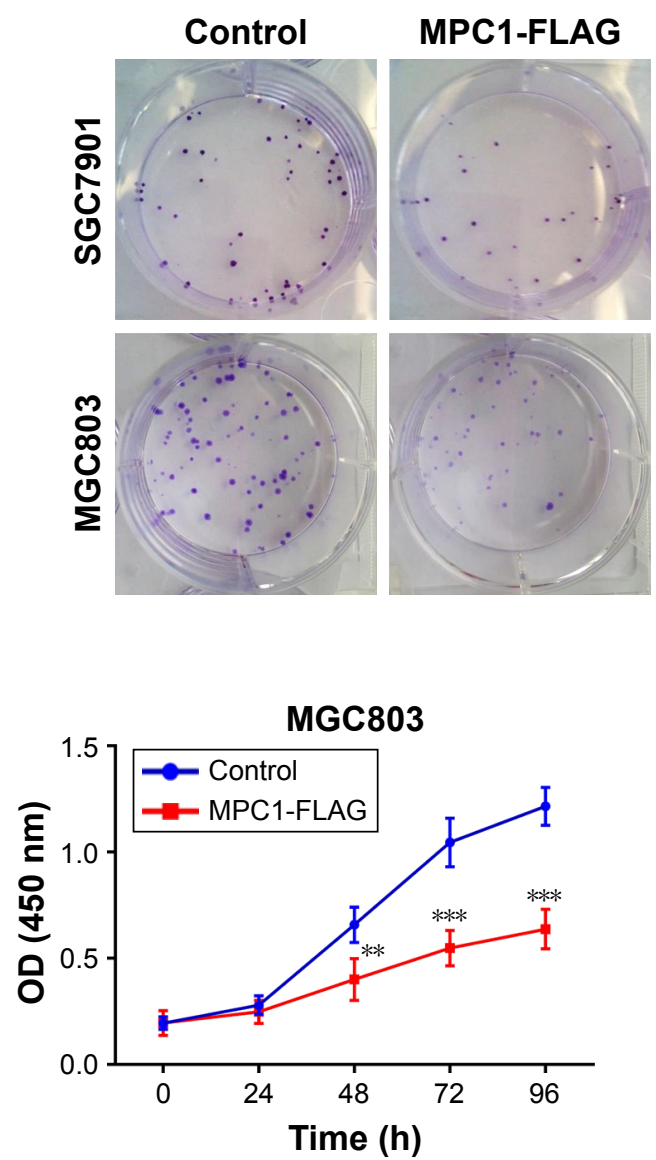

Invasion

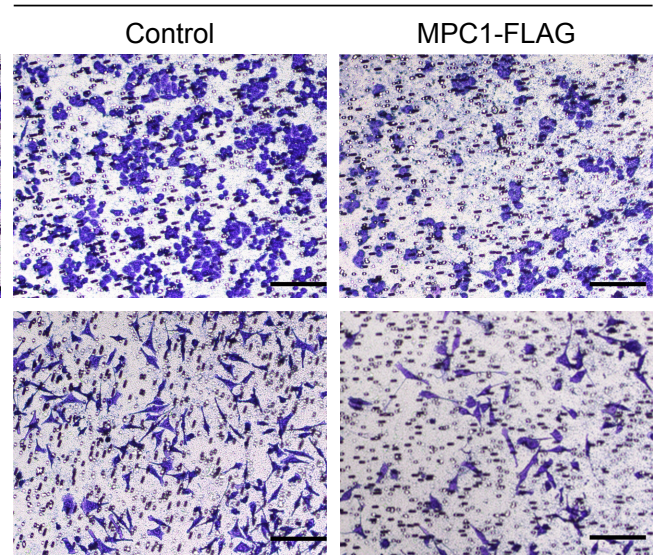

Figure 4 (Continued) 

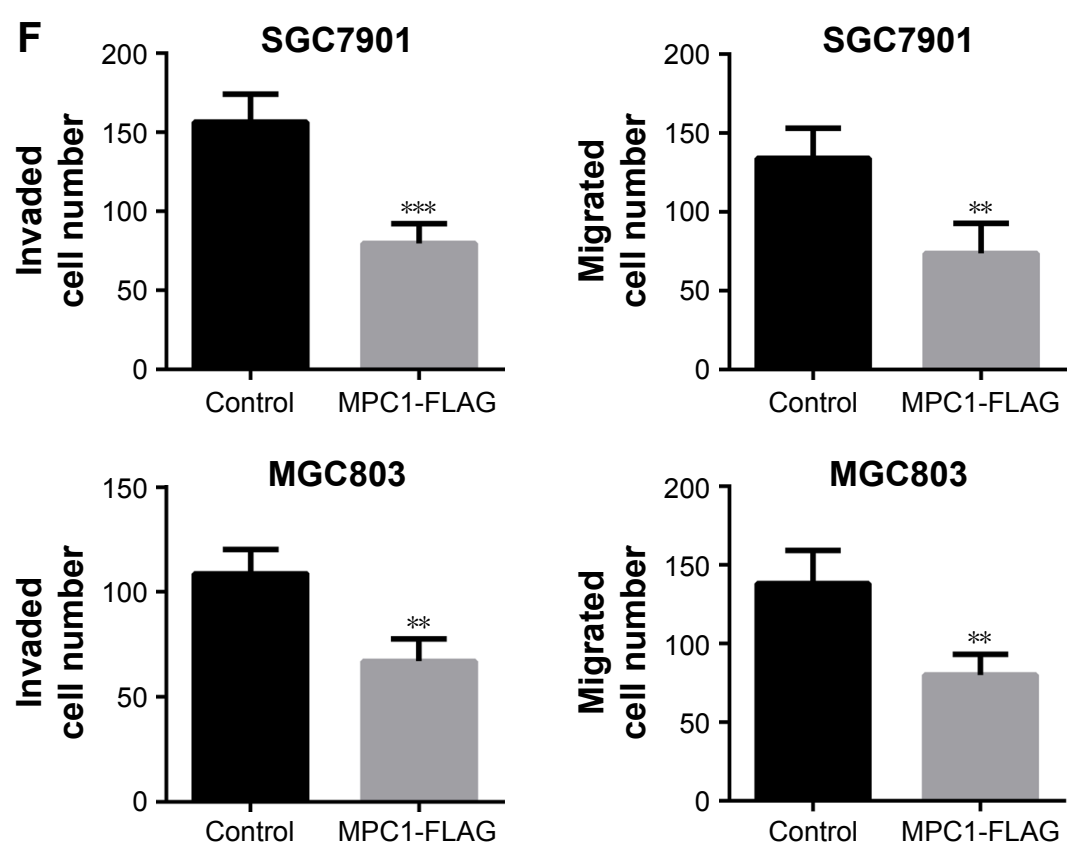

Figure 4 Overexpression of MPCI attenuated the proliferative, migrating, and invasive abilities of human GC cells.

Notes: (A) The overexpression of MPCI in MPCI-FLAG and control GC cells (SGC790I and MGC803) was tested by Western blotting analysis. (B) Representative images of colonies formed in control and MPCI-FLAG GC cells. (C) Histograms showing the number of colonies. The means \pm standard deviations from three independent experiments were shown. (D) The growth rate of control and MPCI-FLAG GC cells (SGC790I and MGC803) was determined by cell counting kit-8 (CCK-8) assay. (E, F) Transwell chamber migration and Matrigel-invasion assays for MPCI-FLAG and control GC cells (SGC790I and MGC803). $* * P<0.01$, $* * * P<0.00 I$. Scale bar, $50 \mu$ m; magnification $\times 200$. Abbreviation: GC, gastric cancer.

formation assay, we found that overexpression of MPC1 resulted in decreased colony number and size in both SGC7901 and MGC803 cells. Our CCK-8 assay demonstrated that overexpression of MPC1 strongly inhibited the proliferation in both cells. In MGC803 cells, significant difference in proliferation was observed as early as $48 \mathrm{~h}$, whereas in SGC7901 cells, it was first observed at $72 \mathrm{~h}$.

Currently, fascinating and novel studies have shown that aerobic glycolysis is also coupled to a series of other cellular functions contributing to the progression of cancer in addition to proliferation. ${ }^{29}$ Tumor cells tend to have increased acid production due to aerobic glycolysis and this resulted in normal cell death and extracellular matrix degradation. These changes facilitate filopodia formation and enhance the migration and invasion of cancer cells. ${ }^{30-34}$ Therefore, we investigated the role of MPC1 in the migrating and invasive capabilities of GC cells. Our results indicated that overexpression of MPC1 significantly decreased the migrating and invasive abilities of GC cells. It was discovered that the inhibition of MPC function by using UK5099 promoted the "stemness" of prostate cancer cells. ${ }^{12}$ In the murine prostate cancer cell line RM-1, MPC1 gene knockout significantly increased "stemness" features, migrating capabilities, and resistance to both chemotherapy and radiotherapy. ${ }^{22}$ Furthermore, it was also reported
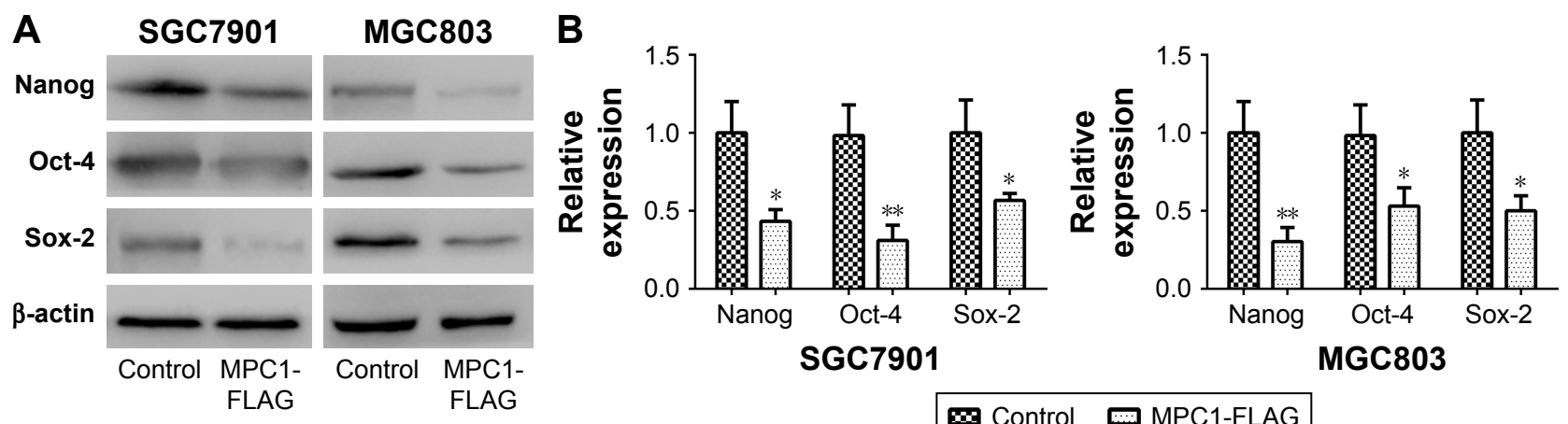

Figure 5 (Continued) 


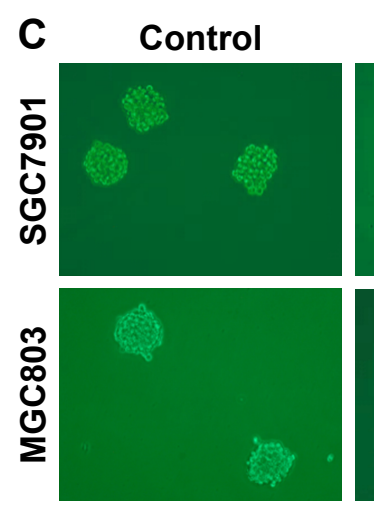

MPC1-FLAG

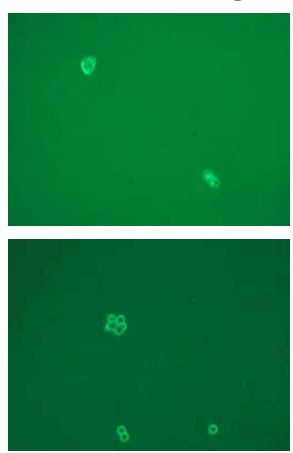

E

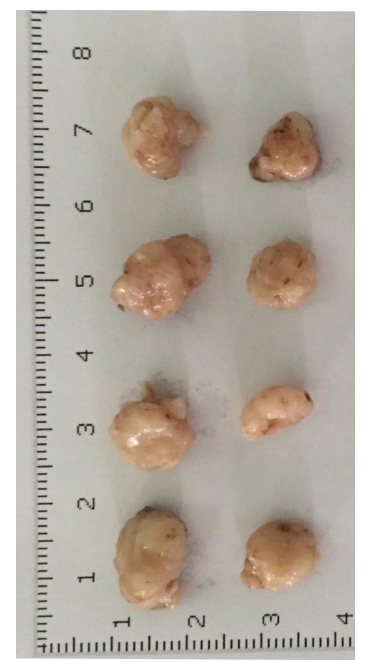

Control MPC1-FLAG

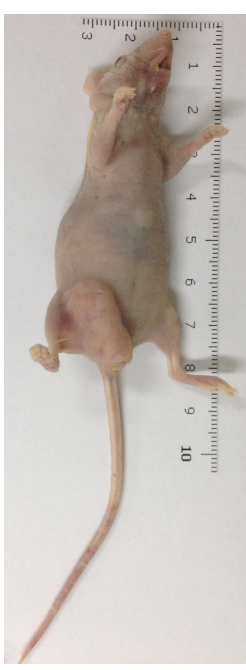

Control

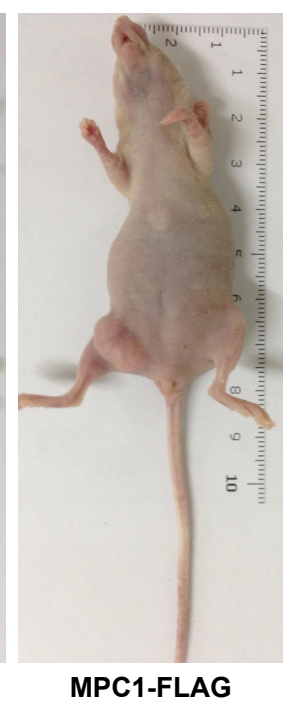

D

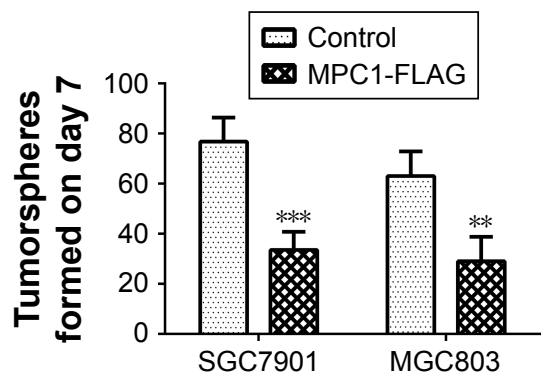

$\mathbf{F}$

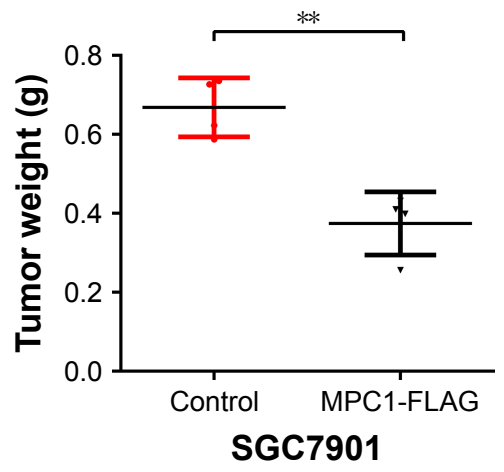

Figure 5 Overexpression of MPCI attenuated the stem cell-like properties of human GC cells.

Notes: (A) Protein levels of "stemness" markers in MPCI-FLAG and control GC cells (SGC790I and MGC803). (B) mRNA levels of "stemness" markers in MPCI-FLAG and control GC cells (SGC790I and MGC803). (C, D) Representative images and quantitative analysis showed that MPCI-FLAG GC cells (SGC790I and MGC803) formed fewer tumorspheres. (E) Xenograft tumors formed by MPCI-FLAG and control GC cells. (F) Analysis of the weight of $x$ enograft tumors. $* P<0.05$, $* * P<0.0 \mathrm{I}$, $* * * P<0.00 \mathrm{I}$. Scale bar, $50 \mu \mathrm{m}$; magnification $\times 200$.

Abbreviation: GC, gastric cancer.

that overexpression of MPC1 in colorectal cancer cell lines resulted in decreased ability of tumorsphere formation, impaired in vivo tumorigenicity, and downregulated stem cell markers. ${ }^{15}$ Thus, we investigated whether overexpression of MPC1 could have an effect on the stem cell properties in GC cells. We selected Nanog, Sox-2, and Oct-4 as GC stem markers. ${ }^{21}$ As shown in Figure 5A and B, overexpression of MPC1 decreased the expression of GC stem markers. Furthermore, we found that MPC1 overexpression inhibited the formation of tumorspheres in vitro and tumor-initiating capacity in vivo. Our study indicated that MPC1 overexpression impaired the stem cell-like properties of GC cells.

\section{Conclusion}

In conclusion, our study illustrates an important function of MPC1 in the malignant behaviors of GC. We demonstrated that MPC1 expression was downregulated in both GC tissues and cell lines. Low MPC1 expression correlates with poor overall survival in patients with GC. Furthermore, MPC1 overexpression inhibits the proliferation, migration, invasion, and stem cell-like properties of GC cells. Our findings suggest that MPC1 is a novel prognostic marker and tumor suppressor gene for patients with GC, and can be a potential candidate target for the treatment of GC.

\section{Acknowledgment}

This work was supported by Science and Technique Development Fund of Sichuan Province (No 140105).

\section{Disclosure}

The authors report no conflicts of interest in this work.

\section{References}

1. Ferlay J, Soerjomataram I, Dikshit R, et al. Cancer incidence and mortality worldwide: sources, methods and major patterns in GLOBOCAN 2012. Int J Cancer. 2015;136(5):E359-E386. 
2. Tan AS, Baty JW, Dong LF, et al. Mitochondrial genome acquisition restores respiratory function and tumorigenic potential of cancer cells without mitochondrial DNA. Cell Metab. 2015;21(1):81-94.

3. Viale A, Pettazzoni P, Lyssiotis CA, et al. Oncogene ablation-resistant pancreatic cancer cells depend on mitochondrial function. Nature. 2014;514(7524):628-632.

4. LeBleu VS, O'Connell JT, Gonzalez HK, et al. PGC-1alpha mediates mitochondrial biogenesis and oxidative phosphorylation in cancer cells to promote metastasis. Nat Cell Biol. 2014;16(10):992-1003.

5. Sancho P, Burgos-Ramos E, Tavera A, et al. MYC/PGC-1alpha balance determines the metabolic phenotype and plasticity of pancreatic cancer stem cells. Cell Metab. 2015;22(4):590-605.

6. Camarda R, Zhou AY, Kohnz RA, et al. Inhibition of fatty acid oxidation as a therapy for MYC-overexpressing triple-negative breast cancer Nat Med. 2016;22(4):427-432.

7. Bricker DK, Taylor EB, Schell JC, et al. A mitochondrial pyruvate carrier required for pyruvate uptake in yeast, Drosophila, and humans. Science. 2012;337(6090):96-100.

8. Herzig S, Raemy E, Montessuit S, et al. Identification and functional expression of the mitochondrial pyruvate carrier. Science. 2012;337(6090): 93-96.

9. Compan V, Pierredon S, Vanderperre B, et al. Monitoring mitochondrial pyruvate carrier activity in real time using a BRET-based biosensor: investigation of the Warburg effect. Mol Cell. 2015;59(3):491-501.

10. Yang C, Ko B, Hensley CT, et al. Glutamine oxidation maintains the TCA cycle and cell survival during impaired mitochondrial pyruvate transport. Mol Cell. 2014;56(3):414-424.

11. Vadvalkar SS, Matsuzaki S, Eyster CA, et al. Decreased mitochondrial pyruvate transport activity in the diabetic heart: role of mitochondrial pyruvate carrier 2 (MPC2) acetylation. J Biol Chem. 2017;292(11): 4423-4433.

12. Zhong Y, Li X, Yu D, et al. Application of mitochondrial pyruvate carrier blocker UK5099 creates metabolic reprogram and greater stem-like properties in LnCap prostate cancer cells in vitro. Oncotarget. 2015 6(35):37758-37769.

13. Li X, Ji Y, Han G, et al. MPC1 and MPC2 expressions are associated with favorable clinical outcomes in prostate cancer. BMC Cancer. 2016; 16(1):894.

14. Wang L, Xu M, Qin J, Lin SC, Lee HJ, Tsai SY, Tsai MJ. MPC1, a key gene in cancer metabolism, is regulated by COUPTFII in human prostate cancer. Oncotarget. 2016;7(12):14673-14683.

15. Schell JC, Olson KA, Jiang L, et al. A role for the mitochondrial pyruvate carrier as a repressor of the Warburg effect and colon cancer cell growth. Mol Cell. 2014;56(3):400-413.

16. Li Y, Li X, Kan Q, et al. Mitochondrial pyruvate carrier function is negatively linked to Warburg phenotype in vitro and malignant features in esophageal squamous cell carcinomas. Oncotarget. 2017;8(1): 1058-1073.

17. Edge SB, Compton CC. The American Joint Committee on Cancer: the 7th edition of the AJCC cancer staging manual and the future of TNM Ann Surg Oncol. 2010;17(6):1471-1474.
18. Shi H, Chen S, Jin H, et al. Downregulation of MSP58 inhibits growth of human colorectal cancer cells via regulation of the cyclin D1-cyclin-dependent kinase 4-p21 pathway. Cancer Sci. 2009;100(9): 1585-1590.

19. Camp RL, Dolled-Filhart M, Rimm DL. X-tile: a new bio-informatics tool for biomarker assessment and outcome-based cut-point optimization. Clin Cancer Res. 2004;10(21):7252-7259.

20. Gentleman RC, Carey VJ, Bates DM, et al. Bioconductor: open software development for computational biology and bioinformatics. Genome Biol. 2004;5(10):R80.

21. Akhavan-Niaki H, Samadani AA. Molecular insight in gastric cancer induction: an overview of cancer stemness genes. Cell Biochem Biophys. 2014;68(3):463-473.

22. Li X, Han G, Li X, et al. Mitochondrial pyruvate carrier function determines cell stemness and metabolic reprogramming in cancer cells Oncotarget. 2017;8(28):46363-46380.

23. Yuan LW, Yamashita H, Seto Y. Glucose metabolism in gastric cancer: The cutting-edge. World J Gastroenterol. 2016;22(6):2046-2059.

24. Cavalli LR, Varella-Garcia M, Liang BC. Diminished tumorigenic phenotype after depletion of mitochondrial DNA. Cell Growth Differ. 1997;8(11):1189-1198.

25. Vander Heiden MG, Cantley LC, Thompson CB. Understanding the Warburg effect: the metabolic requirements of cell proliferation. Science. 2009;324(5930):1029-1033.

26. Eboli ML, Paradies G, Galeotti T, Papa S. Pyruvate transport in tumourcell mitochondria. Biochim Biophys Acta. 1977;460(1):183-187.

27. Schell JC, Rutter J. The long and winding road to the mitochondrial pyruvate carrier. Cancer Metab. 2013;1(1):6.

28. Zhu H, Luo H, Zhu X, Hu X, Zheng L, Zhu X. Pyruvate kinase M2 (PKM2) expression correlates with prognosis in solid cancers: a metaanalysis. Oncotarget. 2017;8(1):1628-1640.

29. Bettum IJ, Gorad SS, Barkovskaya A, et al. Metabolic reprogramming supports the invasive phenotype in malignant melanoma. Cancer Lett. 2015;366(1):71-83.

30. Liu JJ, Liu JY, Chen J, et al. Scinderin promotes the invasion and metastasis of gastric cancer cells and predicts the outcome of patients. Cancer Lett. 2016;376(1):110-117.

31. Lardner A. The effects of extracellular $\mathrm{pH}$ on immune function. J Leukoc Biol. 2001;69(4):522-530.

32. Williams AC, Collard TJ, Paraskeva C. An acidic environment leads to p53 dependent induction of apoptosis in human adenoma and carcinoma cell lines: implications for clonal selection during colorectal carcinogenesis. Oncogene. 1999;18(21):3199-3204.

33. Gatenby RA, Gawlinski ET. The glycolytic phenotype in carcinogenesis and tumor invasion: insights through mathematical models. Cancer Res. 2003;63(14):3847-3854

34. Ward PS, Thompson CB. Metabolic reprogramming: a cancer hallmark even warburg did not anticipate. Cancer Cell. 2012;21(3):297-308. 


\section{Supplementary materials}

Overexpression of $\mathrm{MPCl}$ inhibits the proliferation, migration, invasion, and stem cell-like properties of gastric cancer cells

Table SI Clinical features of patients with GC

\begin{tabular}{lll}
\hline Clinical characteristic & Number & Percentage (\%) \\
\hline Age & & \\
$\geq 60$ & 60 & 39.47 \\
$<60$ & 92 & 60.53 \\
Sex & & \\
Male & 100 & 65.79 \\
Female & 52 & 34.21 \\
Histology grade & & \\
GI & 6 & 3.95 \\
G2 & 37 & 24.34 \\
G3 & 109 & 71.71 \\
T stage & & \\
TI & 31 & 20.39 \\
T2 & 18 & 11.84 \\
T3 & 74 & 48.68 \\
T4 & 29 & 19.08 \\
N stage & & \\
N0 & 61 & 40.13 \\
NI & 41 & 26.97 \\
N2 & 38 & 25.00 \\
N3 & 12 & 7.89 \\
TNM stage & & \\
I & 39 & 25.66 \\
II & 50 & 32.89 \\
III & 47 & 30.92 \\
IV & 16 & 10.53 \\
\hline Abbrevatins & & \\
\hline & &
\end{tabular}

Abbreviations: GC, gastric cancer; TNM, tumor node metastasis.
A

H\&E staining of

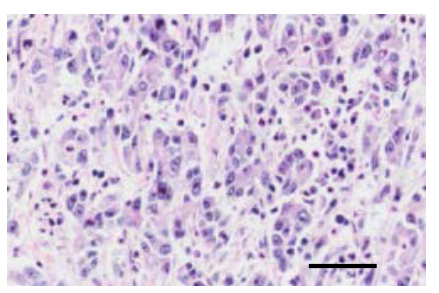

B

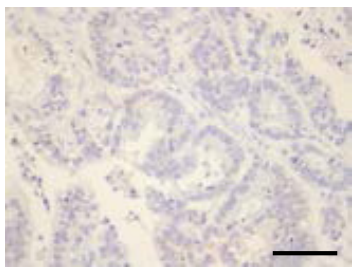

Negative

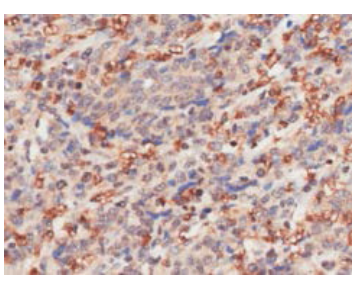

Moderate

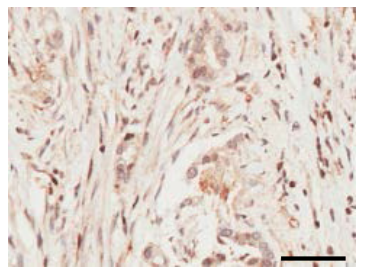

Low

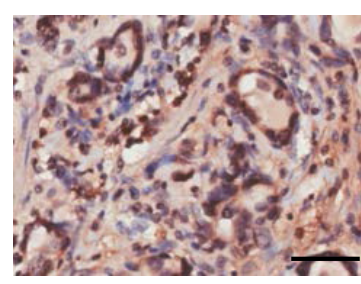

High

\section{Cutoff value for 152 patients with} GC in our cohort
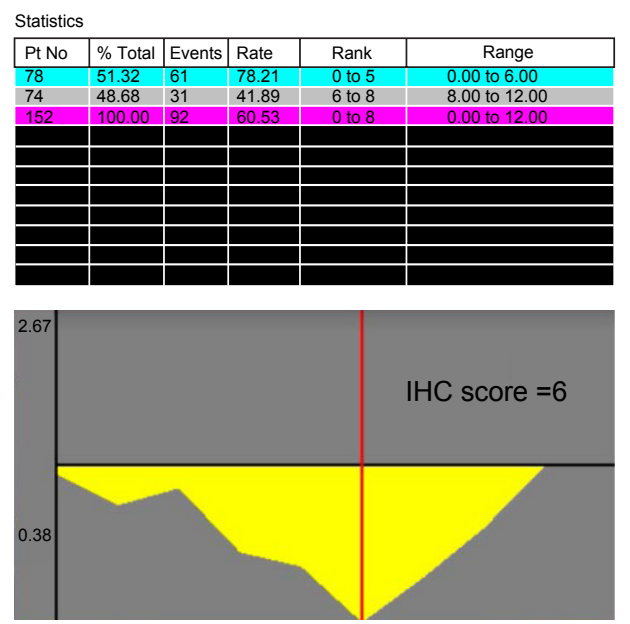

Figure SI (A) H\&E staining of GC samples. (B) The intensity of MPCI in GC cells. Scale bar, $50 \mu \mathrm{m}$; magnification $\times 200$. (C) The relative risk plot of each IHC score generated by $\mathrm{X}$-tile software to identify the optimal cutoff value.

Abbreviations: GC, gastric cancer; IHC, immunohistochemistry; Pt, patient.

Table S2 Sequences of primers used for qRT-PCR in this study

\begin{tabular}{|c|c|c|}
\hline Gene & Forward & Reverse \\
\hline $\mathrm{MPCl}$ & 5'-TCAGTGGGCGGATGACATTTG-3' & 5'-CAGATGCCGTTTTAAGTCATCTCG-3' \\
\hline Nanog & 5'-TGAACCTCAGCTACAAACAG-3' & 5'-TGGTGGTAGGAAGAGTAAAG-3' \\
\hline Sox-2 & 5'-GTATCAGGAGTTGTCAAGGCAGAG & 5'-TCCTAGTCTTAAAGAGGCAGCAAAC-3' \\
\hline Oct-4 & 5'-AGCGAACCAGTATCGAGAAC-3' & 5'-TTACAGAACCACACTCGGAC-3' \\
\hline$\beta$-actin & 5'-TTGCGTTACACCCTTTCTTG-3' & 5'-CACCTTCACCGTTCCAGTTT-3' \\
\hline
\end{tabular}

Abbreviation: qRT-PCR, quantitative reverse transcription polymerase chain reaction. 
OncoTargets and Therapy

\section{Publish your work in this journal}

OncoTargets and Therapy is an international, peer-reviewed, open access journal focusing on the pathological basis of all cancers, potential targets for therapy and treatment protocols employed to improve the management of cancer patients. The journal also focuses on the impact of management programs and new therapeutic agents and protocols on The manuscript management system is completely online and includes a very quick and fair peer-review system, which is all easy to use. Visit http://www.dovepress.com/testimonials.php to read real quotes from published authors.

Submit your manuscript here: http://www.dovepress.com/oncotargets-and-therapy-journal 